\title{
Geometry-induced rectification for an active object
}

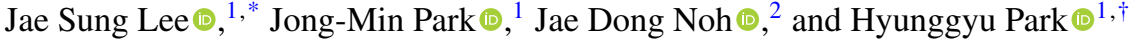 \\ ${ }^{1}$ School of Physics, Korea Institute for Advanced Study, Seoul 02455, Korea \\ ${ }^{2}$ Department of Physics, University of Seoul, Seoul 02504, Korea
}

(Received 12 April 2021; accepted 18 October 2021; published 29 October 2021)

\begin{abstract}
Studies of the rectified current induced by active particles have received great attention due to its possible application to a microscopic motor in biological environments. Insertion of an asymmetric passive object amid many active particles has been regarded as an essential ingredient for generating such a rectified motion. Here, we report that the reverse situation is also possible, where the motion of an active object can be rectified by its geometric asymmetry amid many passive particles. This may describe a unidirectional motion of apolar biological agents with asymmetric shape. We also find a weak but less dispersive rectified motion in a passive mode without energy pump-in. This "moving by dissipation" mechanism could be used as a design principle for developing more reliable microscopic motors.
\end{abstract}

DOI: 10.1103/PhysRevResearch.3.L042011

Introduction. Most biological systems are active in that they are self-propelled, i.e., driven by mechanical forces generated via an internal mechanism consuming chemical fuels [1]. This is in marked contrast to passive systems such as a Brownian particle, whose stochastic motions are governed by external reservoirs. The activeness leads to various unique features clearly distinguished from those of passive systems. For example, active systems show time-scale-dependent diffusivity due to colored noise [2-6], aggregation due to repulsive force [7], efficiency enhancement [8-10], and unconventional entropy production [10-14].

In much of the literature, this self-propelled motion has been encoded into velocity-dependent driving forces $\boldsymbol{F}_{\mathrm{drv}}=$ $-\nabla_{v} \Phi(v)$ at the phenomenological level, where $v$ is the velocity of an active particle and $\Phi(v)$ is a symmetric velocity potential. Specific examples are the Rayleigh-Helmholtz (RH) [15-17], depot [18-21], and Schienbein-Gruler (SG) models $[22,23]$ proposed to describe the driving forces of various active systems such as molecular motors, bacteria, and moving cells, respectively. For each case, the driving force $\boldsymbol{F}_{\mathrm{drv}}$ is given by

$$
\boldsymbol{F}_{\mathrm{drv}}=-\Gamma(\boldsymbol{v}) \boldsymbol{v}, \quad \text { with } \Gamma(\boldsymbol{v})= \begin{cases}\hat{\gamma}+\omega \boldsymbol{v}^{2} & \mathrm{RH} \\ \hat{\gamma} & \text { depot } \\ \hat{\gamma} v_{0} /|\boldsymbol{v}| & \mathrm{SG},\end{cases}
$$

where $v_{0}$ is a velocity unit defined in Eq. (5) and $\hat{\gamma}, \omega$, and $\zeta$ are tunable parameters. Here, we take $\omega, \zeta>0$ for

\footnotetext{
*jslee@kias.re.kr

†hgpark@kias.re.kr

Published by the American Physical Society under the terms of the Creative Commons Attribution 4.0 International license. Further distribution of this work must maintain attribution to the author(s) and the published article's title, journal citation, and DOI.
}

the stability, while $\hat{\gamma}$ can be any real number. When $\boldsymbol{F}_{\text {drv }}$ is applied to a particle with mass $M$, immersed in a liquid with friction coefficient $\gamma>0$ and temperature $T$, the steady-state velocity distribution is given by the exponential form $\sim \exp \left[-M \tilde{\Phi}(v) /\left(k_{\mathrm{B}} T \gamma\right)\right]$ with the effective potential $\tilde{\Phi}(\boldsymbol{v}) \equiv \Phi(\boldsymbol{v})+\gamma \boldsymbol{v}^{2} / 2$. The driving force is denoted to be in the active (passive) mode when $\tilde{\Phi}(\boldsymbol{v})$ is minimum at $\boldsymbol{v} \neq 0$ $(v=0)[1]$. In one dimension, the velocity distribution has symmetric double peaks in the active mode as shown by the left red curve in Fig. 1(a). These two peaks represent a nonzero self-propelling velocity. This active mode appears for $\hat{\gamma}<\gamma_{\mathrm{c}}$, where $\gamma_{\mathrm{c}}=-\gamma$ for the RH and the depot models and $\gamma_{\mathrm{c}}=0$ for the SG model. For $\hat{\gamma}>\gamma_{\mathrm{c}}$, the velocity distribution becomes unimodal with no finite self-propelling velocity [see Fig. 1(a)], which indicates the passive mode. Note that the system is still out of equilibrium even in the passive mode due to the driving force. Even in the active mode, a self-propelled particle does not prefer any particular direction like in a standard run-and-tumble motion [1]; thus there exists no rectified motion (no net particle current) in the long-time limit. The zero current is a natural consequence of the symmetry of the effective potential, $\tilde{\Phi}(\boldsymbol{v})=\tilde{\Phi}(-\boldsymbol{v})$.

In the meantime, the rectification of thermal fluctuations in a Brownian motor has been reported [24,25]. The essential ingredient for the nonzero current of the examples is the interplay of a nonequilibrium condition (temperature gradient) and a geometric asymmetry of the motor. Another interesting example is a "passive" ratchet surrounded by many "active" bacteria, which was found to exhibit a persistent rotational motion (rectified current) in recent experiments [26,27] and numerical simulations [28]. This triggered a flurry of subsequent research [29-35], due to a realistic applicability for designing a microscopic motor in biological environments. Note that this situation also combines nonequilibriumness (activity) and spatial asymmetry of the ratchet.

In this Research Letter, we introduce the reverse situation, where an asymmetric active particle is immersed in a 

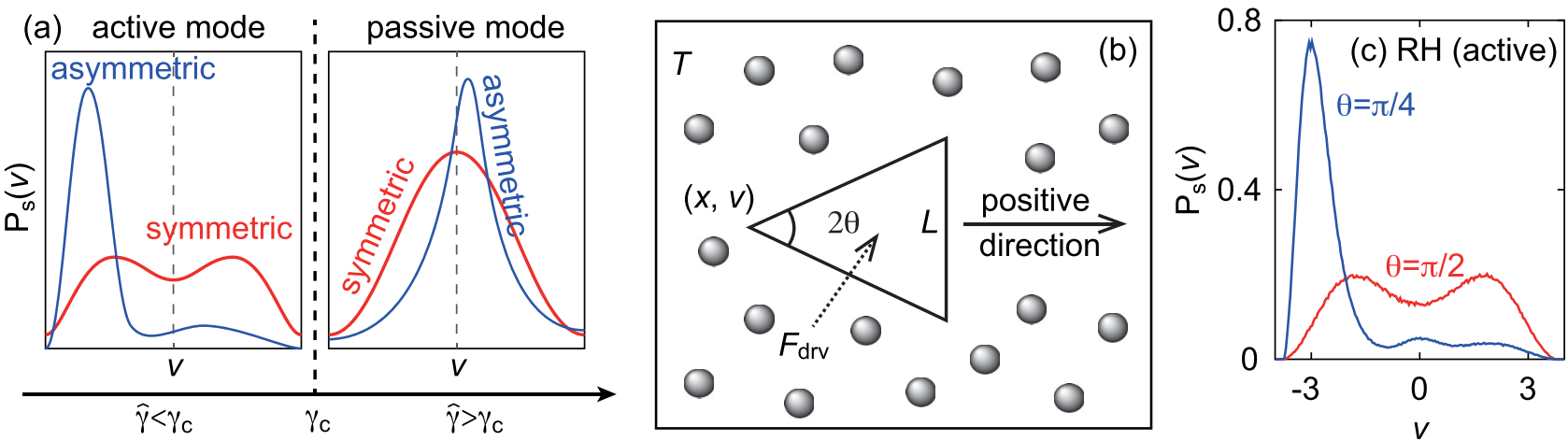

FIG. 1. (a) Schematic of the steady-state velocity distribution $P_{\mathrm{s}}(v)$ in one dimension for driving forces $\mathbf{F}_{\mathrm{drv}}$ in Eq. (1). When $\hat{\gamma}<\gamma_{\mathrm{c}}$ $\left(\hat{\gamma}>\gamma_{\mathrm{c}}\right)$ with a symmetric particle shape, the velocity distribution is symmetric bimodal (unimodal) as shown by the left (right) red curve, which indicates the active (passive) mode. When the shape of a particle is asymmetric, one peak is amplified over the other for the active mode, while the peak position moves slightly aside with reduced variance for the passive mode (see the blue curves). (b) Schematic of the model. The t particle is immersed in a reservoir with temperature $T$ and moves along the horizontal direction (one-dimensional motion). The reservoir consists of $N$ identical $\mathrm{r}$ particles. The driving force $F_{\mathrm{drv}}$ is applied to the t particle. (c) The steady-state velocity distributions for the RH model in the active mode, obtained by numerical simulations. Those for other models and for the passive case are shown in Figs. S1 and S3 of the Supplemental Material [41].

reservoir of passive particles. This problem has been one of the important topics in the active-matter community, but a rigorous theoretical approach has not been addressed to date $[36,37]$. We find that the geometric asymmetry plays a crucial role for the rectified motion along with nonequilibriumness caused by $\boldsymbol{F}_{\text {drv }}$. We derive analytically the explicit formula for the rectified current and compare it with numerical results via extensive molecular dynamics simulations. Interestingly, a rectified current exists even in the passive mode, in particular, for $\hat{\gamma}>0$, where the energy only dissipates (no energy pump-in). We coin the term "moving by dissipation" for this rectifying mechanism when $\hat{\gamma}>0$. In practice, this type of rectification provides a weak but reliable (less dispersive) directed motion even in highly fluctuating thermal environments.

Model. Consider a triangular shaped particle (t particle) with mass $M$ and vertical cross section $L$ immersed in a reservoir with temperature $T$ as illustrated in Fig. 1(b). Here, we constrain the t-particle motion along the horizontal direction only, where its translational motion is essentially equivalent to a rotational motion in the setup for various experimental and theoretical studies [26,27,38,39]. Its apex angle, position, and velocity are denoted as $2 \theta, x$, and $v$, respectively. The reservoir consists of $N$ identical circular shaped particles ( $\mathrm{r}$ particles) with mass $m$ and radius $R$ which move inside a two-dimensional square box with side length $l$ with periodic boundary conditions. We assume that $m \ll M$ and $R \ll L \ll$ $l$. The stochastic motion of the $t$ particle is induced by elastic collisions with $r$ particles, which are equipped with the Langevin thermostat. Details of the collision dynamics and its numerical implementation [24,40] are described in the Supplemental Material [41]. Note that our analysis can be straightforwardly extended to an arbitrary convex shape.

Without any driving force, the $\mathrm{t}$ particle reaches a thermal equilibrium with zero mean velocity. However, with a one-dimensional force in Eq. (1) applied on the t particle, the system can be driven out of equilibrium. The resulting phenomena due to the driving forces are briefly summarized in Fig. 1(a). The asymmetric shape of the $t$ particle in the active mode breaks the symmetry of the peaks as shown by the left blue curve of Fig. 1(a); thus the active motion is rectified with a nonzero average current. This leads to the "rectified run-and-tumble" trajectory for the asymmetric active particle as shown by the blue lines in Fig. S2 of the Supplemental Material [41]. In the passive mode, the asymmetric shape of the $t$ particle moves the position of the unimodal peak slightly aside, representing a weak rectified current compared with that in the active mode as shown by the right blue curve of Fig. 1(a).

Theoretical analysis of the model. To understand the emergence of the nonzero current analytically, we present a perturbation theory for the $\mathrm{t}$ particle dynamics for small $m / M$, which is reasonable in realistic situations. We also assume that $r$ particles are always in equilibrium. Then, our model can be described by the kinetic theory introduced in Refs. [24,25] with the addition of an external driving force.

The probability density of the t particle velocity $v$ at time $t$, $P(v, t)$, can be described by the following Boltzmann master equation:

$$
\frac{\partial P(v, t)}{\partial t}=\left(\mathcal{L}_{\mathrm{res}}+\mathcal{L}_{\mathrm{drv}}\right) P(v, t),
$$

where $\mathcal{L}_{\text {res }}$ and $\mathcal{L}_{\text {drv }}$ are operators representing the effects of the reservoir and the driving force, respectively. More specifically, $\mathcal{L}_{\text {res }}$ can be written as

$$
\mathcal{L}_{\text {res }}=\sum_{n=1}^{\infty} \frac{(-1)^{n}}{n !} \frac{\partial^{n}}{\partial v^{n}} a_{n}(v),
$$

where a Kramers-Moyal coefficient $a_{n}(v)$ is defined as $a_{n}(v) \equiv \int r^{n} W(v+r \mid v) d r$ with the transition rate $W\left(v^{\prime} \mid v\right)$ from $v$ to $v^{\prime}$ induced by elastic collisions with $\mathrm{r}$ particles $[25,42,43]$. The explicit calculation of $a_{n}(v)$ is presented in Sec. II of the Supplemental Material [41]. $\mathcal{L}_{\text {drv }}$ is given by

$$
\mathcal{L}_{\mathrm{drv}}=-\frac{\partial}{\partial v} \frac{F_{\mathrm{drv}}}{M}
$$


with $F_{\mathrm{drv}}=-\Gamma(v) v$ in Eq. (1).

It is convenient to introduce dimensionless variables

$$
v \equiv v / v_{0}\left(v_{0} \equiv \sqrt{k_{\mathrm{B}} T / M}\right) \quad \text { and } \quad \tau=\gamma t / M,
$$

where the $t$ particle friction coefficient $\gamma$ is obtained as

$$
\gamma \equiv 4 L \rho \sqrt{\frac{m k_{\mathrm{B}} T}{2 \pi}}(1+\sin \theta),
$$

with the $\mathrm{r}$ particle density $\rho=N / l^{2}$ (see Sec. II of the Supplemental Material [41]). Then, Eq. (2) is rewritten as

$$
\frac{\partial P(\nu, \tau)}{\partial \tau}=\sum_{n=1}^{\infty} \frac{(-1)^{n}}{n !} \frac{\partial^{n}}{\partial \nu^{n}} A_{n}(\nu) P(\nu, \tau),
$$

where $A_{n}(v)$ is the modified Kramers-Moyal coefficient defined as

$$
A_{n}(v)=\frac{M}{\gamma v_{0}^{n}} a_{n}\left(v_{0} v\right)-G(v) v \delta_{n, 1}
$$

with $P(v, \tau)=v_{0} P(v, t)$ and $G(v)=\Gamma\left(v_{0} v\right) / \gamma$.

We perform a perturbation expansion with the small parameter $\epsilon \equiv \sqrt{m / M}$. Up to $O(\epsilon)$, we find in Sec. II of the Supplemental Material [41]

$$
\begin{aligned}
& A_{1}(v) \approx-[1+G(v)] v+\bar{a}\left(1-v^{2}\right) \epsilon, \\
& A_{2}(v) \approx 2+6 \bar{a} v \epsilon, \\
& A_{3}(v) \approx-12 \bar{a} \epsilon, \\
& A_{n}(v)=O\left(\epsilon^{2}\right), \quad \text { for } n \geqslant 4,
\end{aligned}
$$

where the asymmetric factor $\bar{a}=\frac{\sqrt{2 \pi}}{4}(1-\sin \theta) \geqslant 0$. Using Eqs. (7) and (9) and the expansion of $P(\nu, \tau) \approx P^{(0)}(\nu, \tau)+$ $\epsilon P^{(1)}(\nu, \tau)$, we can set up the equations as follows:

$$
\begin{aligned}
& \partial_{\tau} P^{(0)}(\nu, \tau)=\mathcal{L}_{0} P^{(0)}(\nu, \tau), \\
& \partial_{\tau} P^{(1)}(\nu, \tau)=\mathcal{L}_{1} P^{(0)}(\nu, \tau)+\mathcal{L}_{0} P^{(1)}(\nu, \tau),
\end{aligned}
$$

where $\mathcal{L}_{0}$ and $\mathcal{L}_{1}$ are given by

$$
\begin{aligned}
& \mathcal{L}_{0}=-\frac{\partial}{\partial v}\left[-[1+G(v)] v-\frac{\partial}{\partial v}\right], \\
& \mathcal{L}_{1}=-\bar{a} \frac{\partial}{\partial v}\left[\left(1-v^{2}\right)-3 \frac{\partial}{\partial v} v-2 \frac{\partial^{2}}{\partial v^{2}}\right] .
\end{aligned}
$$

The steady-state distribution of the zeroth order is then

$$
P_{\mathrm{s}}^{(0)}(v)=\frac{1}{\mathcal{N}} e^{-\int^{v} d s[1+G(s)] s}
$$

with the normalization factor $\mathcal{N}$. The next order is obtained by solving $\mathcal{L}_{0} P_{\mathrm{s}}^{(1)}(v)=-\mathcal{L}_{1} P_{\mathrm{s}}^{(0)}(v)$. A straightforward analysis yields

$$
\begin{aligned}
& P_{\mathrm{s}}^{(1)}(v)=\bar{a} g(v) P_{\mathrm{s}}^{(0)}(v) \quad \text { with } \\
& g(v)=2 G(v) v-\int_{0}^{v} d s G(s)[1+2 G(s)] s^{2} .
\end{aligned}
$$

Note that $g(v)$ is an odd function of $v$, i.e., $g(-v)=-g(v)$, as $G(v)$ is even in $v$ in all models considered here. This is also consistent with the normalization condition $\int_{-\infty}^{\infty} d s P_{\mathrm{s}}^{(1)}(s)=$ 0 .
The steady-state average of the $n$th moment of the velocity is then obtained up to $O(\epsilon)$ as

$$
\begin{aligned}
\left\langle v^{n}\right\rangle_{\mathrm{s}} & \approx \int_{-\infty}^{\infty} d v v^{n}[1+\bar{a} \epsilon g(v)] P_{\mathrm{s}}^{(0)}(v) \\
& = \begin{cases}\bar{a} \epsilon\left\langle v^{n} g(v)\right\rangle_{0} & (n \text { odd }) \\
\left\langle v^{n}\right\rangle_{0} & (n \text { even }),\end{cases}
\end{aligned}
$$

where $\langle\cdots\rangle_{0}$ stands for the average over $P_{\mathrm{s}}^{(0)}(v)$. Note that the average velocity and its all-odd moments are $O(\epsilon)$ and vanish for the symmetric case $(\bar{a}=0)$. Thus the time-reversal symmetry breaking (rectified current) occurs only with a shape asymmetry and a finite mass ratio. All even moments responsible for the stochasticity are always $O(1)$, the same as that for $\bar{a}=0$. The standard fluctuation is simply given by the second moment; $\left\langle(\Delta v)^{2}\right\rangle_{s}=\left\langle v^{2}\right\rangle_{s}-\langle v\rangle_{s}^{2} \approx\left\langle v^{2}\right\rangle_{0}$, up to $O(\epsilon)$. Equation (14) can be extended to a particle with arbitrary convex shape with the general expression of $\bar{a}$ and $\gamma$ (see Eqs. (S17) and (S22) in Secs. II and III of the Supplemental Material [41]). This indicates that the rectified motion is a general phenomenon for an active particle with asymmetric shape.

Examples. We first apply the analytic theory to a simple soluble example with $F_{\mathrm{drv}}=-\hat{\gamma} v$ to gain some insight. This model describes a "cold damping" problem applicable to a molecular refrigerator [44-46]. With Eqs. (12)-(14), we find

$$
\langle v\rangle_{\mathrm{s}}^{\mathrm{sim}}=\bar{a} \varepsilon \frac{\hat{\gamma} / \gamma}{(1+\hat{\gamma} / \gamma)^{2}}, \quad\left\langle v^{2}\right\rangle_{\mathrm{s}}^{\mathrm{sim}}=\frac{1}{1+\hat{\gamma} / \gamma}
$$

with $P_{\mathrm{s}}^{(0)}(v) \sim \exp \left[-(1+\hat{\gamma} / \gamma) v^{2} / 2\right]$ (see Sec. III of the Supplemental Material [41]). As expected, the rectification occurs as a result of the interplay of nonequilibrium driving $(\hat{\gamma})$ and spatial asymmetry $(\bar{a})$, even though the driving force does not favor any particular spatial direction. In this simple model, only the passive mode $(\hat{\gamma}>-\gamma)$ is allowed due to the dynamic instability in the active region. We get a nonzero current even when $\hat{\gamma}>0$ without energy input by the driving force. Thus this motion can be called "moving by dissipation" (mbd) denoting that unidirectional motion is induced by purely dissipative force. Note that the $\mathrm{t}$ particle can move in either direction, depending on the sign of $\hat{\gamma}$, and slows down for large positive $\hat{\gamma}$.

Now, we consider the more realistic models for active dynamics given by Eq. (1). We calculated the steady-state velocities $\langle v\rangle_{\mathrm{s}}^{\mathrm{RH}},\langle v\rangle_{\mathrm{s}}^{\mathrm{dpt}}$, and $\langle v\rangle_{\mathrm{s}}^{\mathrm{SG}}$ and their fluctuations for the $\mathrm{RH}$, the depot, and the SG models, respectively. The resulting expressions are rather complex and are presented in Sec. III of the Supplemental Material [41]. The velocities are plotted as solid curves in Fig. 2, which show that current amplitude is much bigger in the stabilized active mode $(\hat{\gamma} / \gamma<-1$ for the RH and the depot models, and $\hat{\gamma}<0$ for the SG model) than in the passive mode. In fact, $\left|\langle v\rangle_{\mathrm{s}}\right|$ grows indefinitely as $\hat{\gamma} \rightarrow-\infty$, and its fluctuation also diverges. In the passive mode, we find a weaker positive current for the $\mathrm{RH}$ and the depot models as observed in the above simple example for $\hat{\gamma}>0$. In contrast, the SG model shows an interesting crossover behavior from a positive to a negative current for positive $\hat{\gamma}$ with $\langle v\rangle_{\mathrm{S}}^{\mathrm{SG}}=-2 \bar{a} \epsilon$ in the $\hat{\gamma} \rightarrow \infty$ limit. Moreover, the fluctuation magnitude of the SG model decays faster than those of the other models: $\left\langle v^{2}\right\rangle_{\mathrm{s}} \sim \hat{\gamma}^{-2}$ (SG) and $\sim \hat{\gamma}^{-1}$ (RH and depot); see Sec. III and Fig. S8 of the Supplemental 

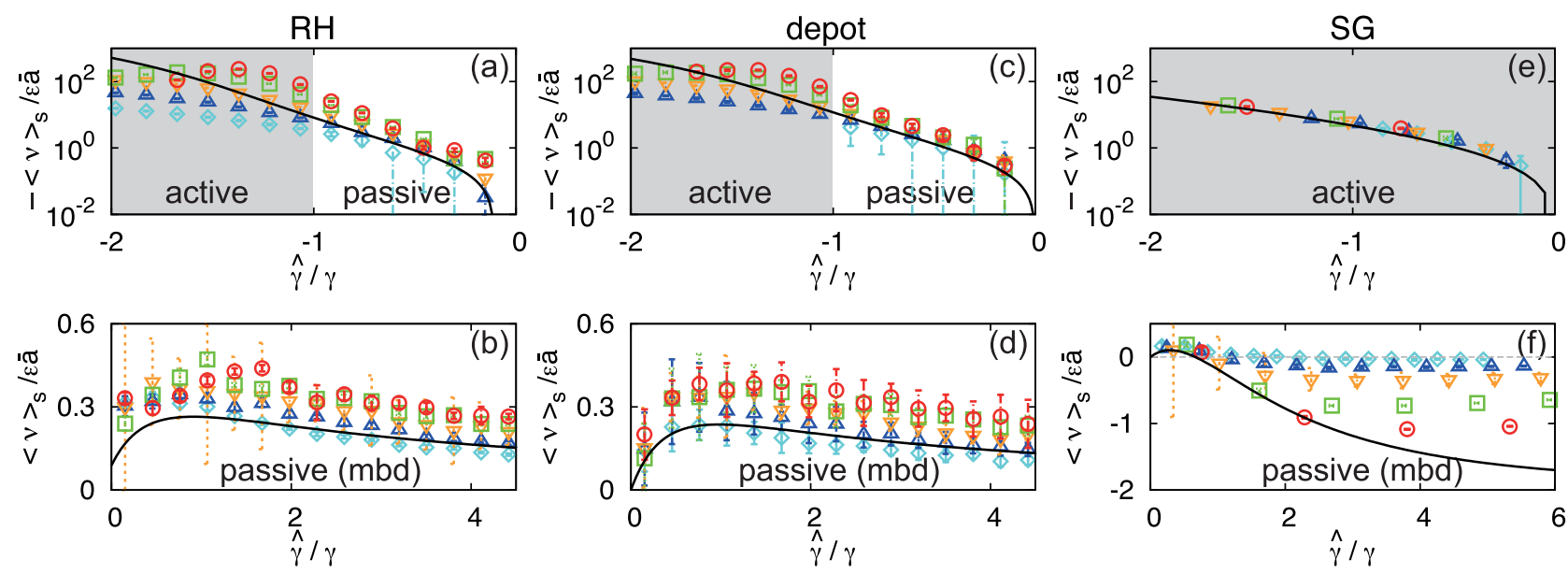

FIG. 2. $\hat{\gamma}$ dependence of the rectified velocity. (a), (c), and (e) are the scaled steady-state velocities $\left|\langle v\rangle_{\mathrm{s}}\right| /(\bar{a} \epsilon)$ as a function of $\hat{\gamma} / \gamma$ for the RH, the depot, and the SG models for negative $\hat{\gamma}$, respectively. (b), (d), and (f) are for positive $\hat{\gamma}$. Solid curves are analytic results. Cyan $\diamond$, blue $\Delta$, gold $\nabla$, green $\square$, and red $\circ$ points denote data for $M=5,10,20,50$, and 100, respectively, with $m=1$ fixed.

Material [41]. Thus the SG type would be more appropriate for designing reliable microscopic motors with both directional currents possible. As a reference, we estimate the order of magnitude of the rectification speed using realistic parameters of physical systems in Sec. IV of the Supplemental Material [41]. Note that this SG case was experimentally studied in Ref. [47].

Numerical simulations. To confirm the validity of our analysis, we performed extensive molecular dynamics simulations. Figure 1(c) shows numerical data for the steady-state velocity distributions of the $t$ particle with a symmetric shape $(\theta=\pi / 2)$ and an asymmetric shape $(\theta=\pi / 4)$ in the active mode for the RH model. The rectification with broken symmetry is clearly seen in the figure. Similar distributions for the depot and the SG models are presented in Fig. S1 of the Supplemental Material [41]. The passive-mode velocity distributions with $\theta=\pi / 4$ and $\hat{\gamma}>0$ are presented in Fig. S3 of the Supplemental Material [41]; they are unimodal and slightly asymmetric as expected.

Data points in Fig. 2 are the simulation results for the rectified velocities of the asymmetric t particle with various values of $\hat{\gamma}$ and t-particle mass $M=5,10,20,50$, and 100 . All other parameters are fixed (see the caption of Fig. S1 of the Supplemental Material [41]). We find that their overall behaviors agree well with the theoretical predictions qualitatively, but with $\sim 40 \%$ overestimates for the simple (Fig. S5 of the Supplemental Material [41]), the RH, and the depot models in the small- $\epsilon$ limit. The origin of this difference is unclear yet, but probably due to a reservoir finite-size effect, also noticed in previous studies in similar systems [24,25]. For the SG model, the numerical overestimate is much smaller, but the convergence to the small $\epsilon$ limit is quite slow for large positive $\hat{\gamma} / \gamma$. This slow convergence is also found in the active mode for the RH and the depot models.

In addition to the emergence of the rectified current, we stress that the geometry-induced current is characterized by suppressed fluctuations in the mbd region. To highlight the usefulness of the mbd mechanism, we compare two simulated trajectories of the same $\mathrm{t}$ particle driven by either constant force $\left(F_{\mathrm{drv}}=f\right)$ or the SG force. The two forces are tuned to yield almost the same average velocity. The blue (red) curve in Fig. 3 is the trajectory averaged over 100 realizations when the $t$ particle is driven by the constant (SG) force. The two trajectories have the same overall slope, but the trajectory from the constant force is much noisier. This strongly suggests that the mbd mechanism can be utilized as a motor mechanism when an accurate motion is required in highly fluctuating environments. However, the rectification speed is quite small as $O(\epsilon)$ in the passive mode; thus its usefulness is rather limited. Note that the origin of the moving by dissipation, motion induced by a geometric asymmetry, is different from that in Refs. [48,49], motion induced by an asymmetric dissipative force.

Conclusion and discussion. Our study clearly demonstrates that the self-propelled motion of an active object can be rectified by its shape asymmetry in passive environments. Our conclusion is applicable to an active object with arbitrary convex shape, driven by a general velocity-dependent force induced by a symmetric velocity potential $\Phi(v)$. We also show that this rectification is possible even in the passive mode without a self-propelled velocity. Especially, for $\hat{\gamma}>$ 0 , the motion is driven by the mbd mechanism, which can provide a design principle for developing more reliable microscopic motors. We note that the active particle motion is also

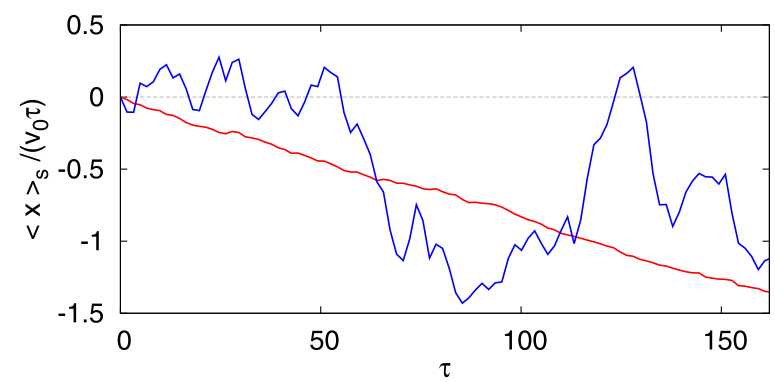

FIG. 3. Trajectories of the $t$ particle averaged over 100 realizations driven by a constant force $f=5.1 \times 10^{-3} \gamma v_{0}$ (blue) and the SG force with $\hat{\gamma} / \gamma=9.88$ (red). $\tau$ has a dimensionless time unit as defined in Eq. (5). 
modeled by introducing a self-propulsion velocity through energy conversions by chemical fuels or reactions $[7,50]$. We find a similar rectification behavior by numerical simulations, shown in Fig. S6 of the Supplemental Material [41].

It is also imaginable that some microorganisms or nanomachines driven by chemical fuels make use of this rectification mechanism by changing their shape asymmetrically to move in an intended direction. Moreover, the magnitude and direction of the $\mathrm{t}$ particle velocity can be controlled by simply changing its shape, i.e., $\theta$ as shown in Fig. S9 of the Supplemental Material [41]. This suggests that the shape change of microorganisms might have an important role in controlling their movement.

Finally, we remark that our result is restricted to onedimensional motion but is still relevant to important problems such as a molecular motor walking along a microtubule, blood cell movement inside a capillary vessel, and a rotary molecu- lar gear. In higher dimensions, the rectification will disappear eventually in the long-time limit due to a possible rotational diffusion, but may generate an intricate interplay between the rotation diffusive time scale and the rectification speed, which is left for future study.

\section{ACKNOWLEDGMENTS}

The authors acknowledge the Korea Institute for Advanced Study for providing computing resources (KIAS Center for Advanced Computation Linux Cluster System). This research was supported by NRF Grants No. 2017R1D1A1B06035497 (H.P.) and No. 2019R1A2C1009628 (J.D.N.) and KIAS Individual Grants No. PG013604 (H.P.), No. PG074002 (J.-M.P.), and No. PG064901 (J.S.L.) at Korea Institute for Advanced Study.
[1] P. Romanczuk, M. Bär, W. Ebeling, B. Lindner, and L. Schimansky-Geier, Active Brownian particles. From individual to collective stochastic dynamics, Eur. Phys. J.: Spec. Top. 202, 1 (2012).

[2] X.-L. Wu and A. Libchaber, Particle Diffusion in a Quasi-TwoDimensional Bacterial Bath, Phys. Rev. Lett. 84, 3017 (2000).

[3] K. C. Leptos, J. S. Guasto, J. P. Gollub, A. I. Pesci, and R. E. Goldstein, Dynamics of Enhanced Tracer Diffusion in Suspensions of Swimming Eukaryotic Microorganisms, Phys. Rev. Lett. 103, 198103 (2009).

[4] J. Palacci, C. Cottin-Bizonne, C. Ybert, and L. Bocquet, Sedimentation and Effective Temperature of Active Colloidal Suspensions, Phys. Rev. Lett. 105, 088304 (2010).

[5] H. Kurtuldu, J. S. Guasto, K. A. Johnson, and J. P. Gollub, Enhancement of biomixing by swimming algal cells in twodimensional films, Proc. Natl. Acad. Sci. USA 108, 10391 (2011).

[6] C. Maggi, M. Paoluzzi, N. Pellicciotta, A. Lepore, L. Angelani, and R. Di Leonardo, Generalized Energy Equipartition in Harmonic Oscillators Driven by Active Baths, Phys. Rev. Lett. 113, 238303 (2014).

[7] Y. Fily and M. C. Marchetti, Athermal Phase Separation of SelfPropelled Particles with No Alignment, Phys. Rev. Lett. 108, 235702 (2012).

[8] S. Krishnamurthy, S. Ghosh, D. Chatterji, R. Ganapathy, and A. K. Sood, A micrometre-sized heat engine operating between bacterial reservoirs, Nat. Phys. 12, 1134 (2016).

[9] R. Zakine, A. Solon, T. Gingrich, and F. van Wijland, Stochastic Stirling engine operating in contact with active baths, Entropy 19, 193 (2017).

[10] J. S. Lee, J.-M. Park, and H. Park, Brownian heat engine with active reservoirs, Phys. Rev. E 102, 032116 (2020).

[11] C. Kwon, J. Yeo, H. K. Lee, and H. Park, Unconventional entropy production in the presence of momentum-dependent forces, J. Korean Phys. Soc. 68, 633 (2016).

[12] H. K. Lee, S. Lahiri, and H. Park, Nonequilibrium steady states in Langevin thermal systems, Phys. Rev. E 96, 022134 (2017).

[13] É. Fodor, C. Nardini, M. E. Cates, J. Tailleur, P. Visco, and F. van Wijland, How Far from Equilibrium Is Active Matter? Phys. Rev. Lett. 117, 038103 (2016).
[14] L. Dabelow, S. Bo, and R. Eichhorn, Irreversibility in Active Matter Systems: Fluctuation Theorem and Mutual Information, Phys. Rev. X 9, 021009 (2019).

[15] M. Badoual, F. Jülicher, and J. Prost, Bidirectional cooperative motion of molecular motors, Proc. Natl. Acad. Sci. USA 99, 6696 (2002).

[16] D. Chaudhuri, Active Brownian particles: Entropy production and fluctuation response, Phys. Rev. E 90, 022131 (2014).

[17] C. Ganguly and D. Chaudhuri, Stochastic thermodynamics of active Brownian particles, Phys. Rev. E 88, 032102 (2013).

[18] F. Schweitzer, W. Ebeling, and B. Tilch, Complex Motion of Brownian Particles with Energy Depots, Phys. Rev. Lett. 80, 5044 (1998).

[19] W. Ebeling, F. Schweitzer, and B. Tilch, Active Brownian particles with energy depots modeling animal mobility, BioSystems 49, 17 (1999).

[20] C. A. Condat, and G. J. Sibona, Diffusion in a model for active Brownian motion, Phys. D (Amsterdam) 168, 235 (2002).

[21] V. Garcia, M. Birbaumer, and F. Schweitzer, Testing an agent-based model of bacterial cell motility: How nutrient concentration affects speed distribution, Eur. Phys. J. B 82, 235 (2011).

[22] M. Schienbein and H. Gruler, Langevin equation, FokkerPlanck equation and cell migration, Bull. Math. Biol. 55, 585 (1993).

[23] U. Erdmann, W. Ebeling, L. Schimansky-Geier, and F. Schweitzer, Brownian particles far from equilibrium, Eur. Phys. J. B 15, 105 (2000).

[24] C. Van den Broeck, R. Kawai, and P. Meurs, Microscopic Analysis of a Thermal Brownian Motor, Phys. Rev. Lett. 93, 090601 (2004).

[25] P. Meurs, C. Van den Broeck, and A. Garcia, Rectification of thermal fluctuations in ideal gases, Phys. Rev. E 70, 051109 (2004).

[26] A. Sokolov, M. M. Apodaca, B. A. Grzybowski, and I. S. Aranson, Swimming bacteria power microscopic gears, Proc. Natl. Acad. Sci. USA 107, 969 (2010).

[27] R. Di Leonardo, L. Angelani, D. Dell'Arciprete, G. Ruocco, V. Iebba, S. Schippa, M. P. Conte, F. Mecarini, F. De Angelis, and 
E. Di Fabrizio, Bacterial ratchet motors, Proc. Natl. Acad. Sci. USA 107, 9541 (2010).

[28] L. Angelani, R. Di Leonardo, and G. Ruocco, Self-Starting Micromotors in a Bacterial Bath, Phys. Rev. Lett. 102, 048104 (2009).

[29] Y. Baek, A. P. Solon, X. Xu, N. Nikola, and Y. Kafri, Generic Long-Range Interactions Between Passive Bodies in an Active Fluid, Phys. Rev. Lett. 120, 058002 (2018).

[30] L. Angelani and R. Di Leonardo, Geometrically biased random walks in bacteria-driven micro-shuttles, New J. Phys. 12, 113017 (2010).

[31] A. Kaiser, A. Peshkov, A. Sokolov, B. ten Hagen, H. Löwen, and I. S. Aranson, Transport Powered by Bacterial Turbulence, Phys. Rev. Lett. 112, 158101 (2014).

[32] N. Nikola, A. P. Solon, Y. Kafri, M. Kardar, J. Tailleur, and R. Voituriez, Active Particles with Soft and Curved Walls: Equation of State, Ratchets, and Instabilities, Phys. Rev. Lett. 117, 098001 (2016).

[33] C. J. O. Reichhardt and C. Reichhardt, Ratchet effects in active matter systems, Annu. Rev. Condens. Matter Phys. 8, 51 (2017).

[34] G. Vizsnyiczai, G. Frangipane, C. Maggi, F. Saglimbeni, S. Bianchi, and R. Di Leonardo, Light controlled 3D micromotors powered by bacteria, Nat. Commun. 8, 15974 (2017).

[35] A. Kaiser, A. Sokolov, I. S. Aranson, and H. Löwen, Motion of two micro-wedges in a turbulent bacterial bath, Eur. Phys. J.: Spec. Top. 224, 1275 (2015).

[36] A. Aubret, M. Youssef, S. Sacanna, and J. Palacci, Targeted assembly and synchronization of self-spinning microgears, Nat. Phys. 14, 1114 (2018).

[37] F. Kümmel, B. ten Hagen, R. Wittkowski, I. Buttinoni, R. Eichhorn, G. Volpe, H. Löwen, and C. Bechinger, Circular
Motion of Asymmetric Self-Propelling Particles, Phys. Rev. Lett. 110, 198302 (2013).

[38] C. Van den Broeck and R. Kawai, Brownian Refrigerator, Phys. Rev. Lett. 96, 210601 (2006).

[39] M. van den Broek and C. Van den Broeck, Chiral Brownian Heat Pump, Phys. Rev. Lett. 100, 130601 (2008).

[40] J. S. Lee and H. Park, Additivity of multiple heat reservoirs in the Langevin equation, Phys. Rev. E 97, 062135 (2018).

[41] See Supplemental Material at http://link.aps.org/supplemental/ 10.1103/PhysRevResearch.3.L042011 for details of numerical simulations and analytic derivations.

[42] H. Risken, The Fokker-Planck Equation (Springer, Berlin, 1989).

[43] N. G. van Kampen, Stochastic Processes in Physics and Chemistry (Elsevier, New York, 2007).

[44] M. Pinard, P. F. Cohadon, T. Briant, and A. Heidmann, Full mechanical characterization of a cold damped mirror, Phys. Rev. A 63, 013808 (2000).

[45] K. H. Kim and H. Qian, Entropy Production of Brownian Macromolecules with Inertia, Phys. Rev. Lett. 93, 120602 (2004).

[46] G. Jourdan, G. Torricelli, J. Chevrier, and F. Comin, Tuning the effective coupling of an AFM lever to a thermal bath, Nanotechnology 18, 475502 (2007).

[47] A. Gnoli, A. Petri, F. Dalton, G. Pontuale, G. Gradenigo, A. Sarracino, and A. Puglisi, Brownian Ratchet in a Thermal Bath Driven by Coulomb Friction, Phys. Rev. Lett. 110, 120601 (2013).

[48] J. Talbot, R. D. Wildman, and P. Viot, Kinetics of a Frictional Granular Motor, Phys. Rev. Lett. 107, 138001 (2011).

[49] R. García-García, P. Collet, and L. Truskinovsky, Guided active particles, Phys. Rev. E 100, 042608 (2019).

[50] S. J. Ebbens and J. R. Howse, In pursuit of propulsion at the nanoscale, Soft Matter 6, 726 (2010). 